\title{
Das kollegiale Gespräch
}

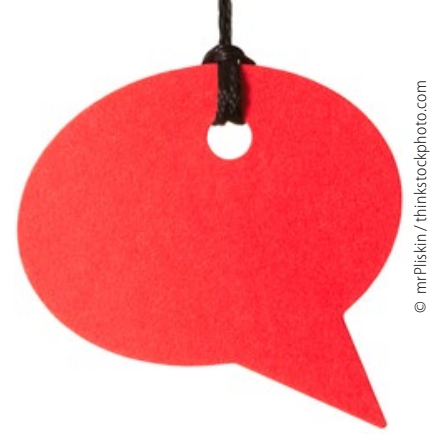

\author{
Die Demografie hat auch die Zahnärzte fest im Griff, \\ und ihre Praxen werden zunehmend mit älteren multi- \\ morbiden, gehandicapten Patienten konfrontiert. Nicht \\ zuletzt deshalb hat der Gemeinsame Bundesausschuss \\ (G-BA) neue Leistungen in den Bewertungsmaßstab \\ zahnärztlicher Leistungen (BEMA) aufgenommen und \\ der Abrechnung zugänglich gemacht.
}

So gut wie jeder Praxisinhaber kann ein Lied davon singen: Gab es früher nur vereinzelte Anfragen von Angehörigen, ob der Zahnarzt auch gehandicapte Patienten oder Pflegefälle behandelt, hat der Bedarf mittlerweile stark zugenommen. Verschiedene Verlage fragen nach behindertengerechten Einrichtungen und Zugängen. Die Standesverwaltung fragt für die digitale Zahnarztsuche nach der Möglichkeit des barrierefreien Zugangs oder gar Hausbesuchen. Die Bundesländer überschlagen sich mit Ankündigungen wie zum Beispiel „Bayern wird barrierefrei“, will das aber für den Freistaat kostenneutral, also auf Kosten der Praxisinhaber, dennoch gefördert durch Sonderkreditprogramme. Und natürlich hat jede Leistungserweiterung im BEMA auch ihre Schattenseiten. Mit dem Aufschlag zur aufsuchenden Betreuung hat man die Morbidität ohne Budgetgrenzen feststellen lassen. Es war ja nur von Besuchen die Rede. Die darauffolgende Behandlung wurde als Bedarf interpretiert, der aber wegen eingeschränkter Behandlungsmöglichkeiten nicht einfach zu decken war.

Zum einen ist eine „Bettkantenzahnmedizin“ rein rechtlich nur unter den gleichen Voraussetzungen wie in der Praxis machbar, was praktisch fast unmöglich ist, da die Vorgaben des Robert Koch-Instituts die Zahnmedizin nahezu mit der Operation am offenen Herzen gleichsetzen. Zum anderen sind die Folgeleistungen, die am Patienten erbracht werden, in den meisten Bundesländern budgetrelevant.

Ein weiteres Kriterium sind Leistungen, die nur bei einem Versorgungsvertrag mit einer Pflegeeinrichtung erbracht und abgerechnet werden können. Auch hier sind beachtliche bürokratische Hürden zu überwinden.

Die Artikelserie „Der gangbare Weg“ beleuchtet die neuen Leistungen in mehreren Artikeln. Als Einstieg werden die sehr wichtigen neuen Positionen BEMA Nr. 181 und 182 erläutert.

\section{Konsiliarische Erörterung mit Ärzten und Zahnärzten (Ksl)}

Die vorgeschriebene Dokumentation muss den Namen des Arztes/Zahnarztes oder der Ärzte/Zahnärzte, den Grund der konsillarischen Erörterung, die Vorgehensweise oder den weiteren Ablauf und gegebenenfalls die Therapieplanung beinhalten. Abrechenbar ist sie je Konsiliar.

Zusätzliche Abrechnungsbestimmungen:

- Die Leistung nach Nr. 181 ist nur abrechnungsfähig, wenn sich der Zahnarzt zuvor oder im unmittelbaren zeitlichen Zusammenhang mit der konsiliarischen Erörterung persönlich mit dem Versicherten und dessen Erkrankung befasst hat.
- Die Leistung nach Nr. 181 ist auch dann abrechnungsfähig, wenn die Erörterung zwischen einem Zahnarzt und dem ständigen persönlichen ärztlichen/zahnärztlichen Vertreter eines anderen Arztes/Zahnarztes erfolgt.

- Die Leistung nach Nr. 181 ist nicht abrechnungsfähig, wenn die Zahnärzte Mitglieder derselben Berufsausübungsgemeinschaft oder einer Praxisgemeinschaft von Ärzten/Zahnärzten gleicher oder ähnlicher Fachrichtung sind. Sie ist nicht abrechnungsfähig für routinemäßige Besprechungen.

\section{Konsiliarische Erörterung mit Ärzten und Zahnärzten im Rahmen eines Kooperationsvertrags nach § 119b Abs. 1 SGB V (KsIK)}

Die vorgeschriebene Dokumentation muss den Namen des Arztes/Zahnarztes bzw. der Ärzte/Zahnärzte, den Grund der konsillarischen Erörterung, die Vorgehensweise beziehungsweise den weiteren Ablauf und gegebenenfalls die Therapieplanung beinhalten. Abrechenbar ist sie je Konsil .

Die Leistung nach Nr. 182 ist nur abrechnungsfähig für konsiliarische Erörterungen, die pflegebedürftige Versicherte betreffen, welche in einer stationären Pflegeeinrichtung ( $\$ 71$ Abs. 2 SGB XI) betreut werden und der Vertragszahnarzt mit der stationären Pflegeeinrichtung einen Kooperationsvertrag gemäß $\$ 119 b$ Abs. 1 SGB $\mathrm{V}$ geschlossen hat, welcher den verbindlichen Anforderungen der Vereinbarung nach $\$ 119 \mathrm{~b}$ Abs. 2 SGB V entspricht, und wenn die hierfür zuständige Kassenzahnärztliche Vereinigung die Berechtigung zur Abrechnung festgestellt hat. Darüber hinaus gelten die Abrechnungsbestimmungen 2 und 3 der BEMA-Nr. 181 ebenfalls.

Gerade das sehr geschätzte kollegiale Gespräch auch zwischen allgemeinärztlichen Kollegen und zahnärztlichen Kollegen wie auch das Gespräch mit dem Kieferchirurgen oder Kieferorthopäden bringen Klarheit in den Behandlungsablauf. Denn was gemeinsam besprochen, geplant und dokumentiert wird, führt stets zu besseren Ergebnissen. Immer komplexere Behandlungen, die steigende Zahl multimorbider Patienten mit immer weniger kalkulierbareren Nebenwirkungen neuer Pharmazeutika machen dies erforderlich. Nicht zu unterschätzen ist die forensische Relevanz nicht stattgefundener Konsiliare. Man bedenke gerade die NOAK (neue orale Antikoagulanzien) oder die in der Krebstherapie ständig steigende Anzahl von Menschen mit Bisphosphonattherapie, die unbeachtet katastrophale Auswirkungen hat.

Zahnärzte sollten die Möglichkeit nutzen, zum Wohle der Patienten interkollegial zu kommunizieren - gerade auch, wenn es doch abrechenbar ist.

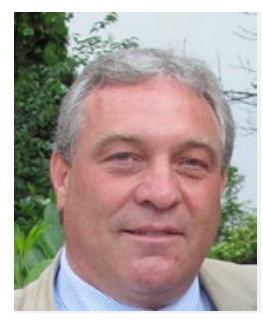

Dr. Christian Öttl

Mitglied im FVDZ-Bundesvorstand 